\title{
Successful use of Eltrombopag in a patient with refractory HIV-associated immune thrombocytopenic purpura
}

\author{
Juárez-Salcedo $\mathrm{LM}^{1+*}$, Conde $\mathrm{D}^{2 \dagger}$, Dalia $\mathrm{S}^{3}$ \\ ${ }^{1}$ Hematology Department, Gregorio Marañón University Hospital, Madrid, Spain \\ ${ }^{2}$ Principe de Asturias University Hospital, Madrid, Spain \\ ${ }^{3}$ Hematology/Oncology Department, Mercy Clinic Oncology and Hematology - Joplin, Missouri, United States \\ ${ }^{\dagger}$ Both authors contributed equally to this manuscript
}

\begin{abstract}
Immune Thrombocytopenic Purpura (ITP) is not uncommon in patients with Human Immunodeficiency Virus (HIV) infection. This entity can be recurrent to standard therapy, which includes corticosteroids, intravenous immunoglobulins (IVIG) and highly active antiretroviral therapy (HAART). In immunocompetent patients with ITP, Eltrombopag, a thrombopoetin receptor agonist (TRA), has proven to be save and useful for increasing platelet counts and for treating patients with Hepatitis $\mathrm{C}$ virus (HCV) associated thrombocytopenia that need an interferon-based therapy. Safety and efficacy data with the use of TRAs in HIV-associated ITP are scarce, being a non-authorized indication. We report our one-year experience with Eltrombopag for treating a severe and refractory HIV-associated ITP.
\end{abstract}

\section{Introduction}

Thrombocytopenia has been associated with HIV since the beginning of the AIDS epidemic [1,2]. Immune thrombocytopenia purpura (ITP) was a relatively common abnormality, which was seen in as many as $30 \%$ of infected patients especially before the HAART era [35]. Nowadays, HIV-associated ITP has reduced the incidence to $3-5 \%$ [6,7]. Both, decreased production of platelets and increased destruction have been proposed as mechanisms of thrombocytopenia. Two different clinical situations may be seen in HIV-associated ITP: patients with advanced AIDS in which thrombocytopenia is a manifestation of bone marrow suppression and patients without significant T-cell depletion in whom thrombocytopenia develops likely as an immune phenomenon. In HIV-associated ITP, the standard treatments for classical ITP (corticosteroids, IVIG, splenectomy, danazol, Anti-D, dapsone and splenic radiation) have all been employed with varying degrees of success, being the highest response rates achieved in the HAART era [8]. When platelet counts remain persistently below $20 \times$ $10^{9} / \mathrm{L}$ despite therapies, patients retain a significantly increased risk of bleeding complications [9].

Eltrombopag, one of the TRAs, was granted approval for the treatment of refractory ITP after the failure of first line therapy, being effective in a high percentage of cases. Therapy with Eltrombopag for refractory HIV-associated ITP has not been sufficiently studied or reported. We report our successful use of Eltrombopag in a patient with HIV-associated ITP who was refractory to standard treatments.

\section{Case report}

A 61-year-old male with a history of morbid obesity, hypertension, coronary artery disease and dyslipidemia, was diagnosed of HIV infection in 2005. He was started on HAART (Abacavir, Lamivudine and Efavirenz), and 6 months after beginning, a non-detectable viral load and CD4 count over 500 cells $/ \mathrm{mm}^{3}$ were both achieved.
In January 2006, he presented with multiple cervical, axillary, abdominal lymphadenopathies and splenomegaly, being diagnosed of Follicular Non-Hodgkin Lymphoma (NHL), grade I, stage IIIA, FLIPI 1. He achieved a complete response (CR) after 8 cycles of Rituximab, Cyclophosphamide, Vincristine and Prednisone, following annual controls in the Hematology department.

In July 2014, while remaining on continuous CR of the NHL, the patient was admitted because of acute generalized petechias on trunk and extremities and hemorrhagic blisters in the oral mucosa. Analytical results at the initial evaluation included hemoglobin $12 \mathrm{~g} /$ $\mathrm{dl}$, hematocrit 37\%, MCV $97.2 \mathrm{fL}, \mathrm{WBC} 7.4 \times 10^{9} / \mathrm{L}$ (neutrophils $5.4 \times$ $10^{9} / \mathrm{L}$, lymphocyte $0.87 \times 10^{9} / \mathrm{L}$ ), and platelets $2 \times 10^{9} / \mathrm{L}$. The patient's chemistry and liver function tests were within normal limits. Additional testing revealed undetectable HIV viral load and CD4 count of 574 cell/ $\mathrm{mm}^{3}$.

Bone Marrow biopsy showed adequate trilineage hematopoiesis and increased megakaryopoiesis in keeping with a peripheral destructive an etiology for the thrombocytopenia.

The patient was initially treated with Prednisone $1 \mathrm{mg} / \mathrm{kg}$ daily plus IVIG $0,4 \mathrm{~g} / \mathrm{Kg}$ for five consecutive days, without initially increasing in the platelet counts. After two weeks, platelets moderately improved to $37 \times 10^{9} / \mathrm{L}$ but spontaneously dropped to $22 \times 10^{9} / \mathrm{L}$, being necessary to repeat a new course of IVIG while maintaining daily corticosteroids treatment. Over the next two months, a high dose of prednisone (100

*Correspondence to: Luis Miguel Juárez Salcedo, Hematology Department, Gregorio Marañón University Hospital, Madrid, Spain, E-mail: dr.luisjuarez@ gmail.com

Key words: Eltrombopag, thrombopoetin receptor agonist, HIV/AIDS, immune thrombocytopenia purpura

Received: April 02, 2020; Accepted: April 12, 2020; Published: April 18, 2020 
mg per day) was impossible to reduce by continuous and difficult to control hyperglycemic episodes, and with a minimal platelets response (platelets count between $7 \times 10^{9} / \mathrm{L}$ and $37 \times 10^{9} / \mathrm{L}$ ). Splenectomy was considered but was avoided due to concern regarding potential infectious complications. After requesting patient's written informed consent, a compassionate use of TRAs was decided. Eltrombopag at a dose of $50 \mathrm{mg}$ p.o daily was initiated as an alternative treatment for refractory HIV-related thrombocytopenia. After 4 weeks, platelets count rose to $485 \times 10^{9} / \mathrm{L}$ allowing the progressive tapering of prednisone until its complete interruption. Currently, 6 months after starting on Eltrombopag, platelets counts are stable between 185 and $220 \times 10^{9} / \mathrm{L}$, with $25 \mathrm{mg}$ twice a week as the minimum effective dose, with no hepatotoxicity signs or history of thrombotic episodes.

\section{Discussion}

Thrombocytopenia (TP) is common in patients with HIV infection, reported in as many as $10-30 \%$ of patients and seen more often in IV drug users and advanced HIV infections even in post-HAART era [8].

The pathophysiology of HIV-associated ITP has not been fully elucidated but is likely somewhat different than that of classic ITP [10]. Severe T-cell depletion and consequent immune dysregulation occurs with HIV infection. In addition, megakaryocytes express HIV receptors CD4, CXCR4 and CCR5, and therefore, may be vulnerable to direct viral cytotoxicity, which ultimately could lead to a decrease in platelets formation [11]. Antiplatelet immune complexes are also present on platelets of HIV-infected patients and may be an important contributor to ITP pathogenesis [11].

The common treatments for classical ITP have been used with varying degrees of success and the highest response rates seen in the HAART era. Shortly after HAART became available, antiretrovirals were shown to be successful in treating ITP in a randomized trial of Zidovudine monotherapy [12]. Long-term treatment with corticosteroids, produce an initial rapid response but induce immunosuppression. IVIG do not have this risk but lack sustained effects. Splenectomy is reserved for resistant HIV-associated thrombocytopenia and is curative in 50 percent of patients [13].

Recently, Kowalczyk and colleagues, described 5 patients with refractory HIV-related ITP. These patients, unlike our patient, were diagnosed of HIV at the same time of the ITP symptoms appearance. All 5 patients had failed or relapsed after conventional treatment (corticosteroids and IVIG) and recovered the platelets counts after received TRAs. Our patient presented the ITP episode after 10 years on HAART, not being in relationship with HIV- progression, documented infection or daily medication changes. NHL relapsing disease was also excluded. As many reported cases, the initial therapy option was full-dose corticosteroids plus five days of IVIG without an adequate platelet response and severe secondary metabolic effects. After starting treatment with TRAs, our patient presented platelets count recovery.

TRAs have been granted approval for the treatment of refractory ITP. These agents are effective in between $59 \%$ to $88 \%$ of ITP cases, and loss of response while on continued therapy is uncommon $[14,15]$. Eltrombopag, an oral, non-peptide TRA that interacts with thrombopoietin receptor leading to JAK/STAT pathway signaling in megakaryocytes, has been well studied in several clinical trials evaluating their effectiveness in treating various subsets of patients with problematic ITP. However, these studies have not detailed its use and efficacy in the treatment of HIV-associated thrombocytopenia. For this kind of patients, there is a substantial need for additional treatment options beyond the traditional ITP therapies, as platelet counts often remain inadequate despite multiple interventions. Side effects associated with TRAs are well documented. An increase in bone marrow reticulin fibrosis has been reported in $5 \%$ of patients, but to date there are no disconcerting signals to suggest that the marrow fibrosis is progressive or irreversible [16,17]. Eltrombopag, also carries a warning because of increased risk of hepatotoxicity. Additionally, portal vein thrombosis has been reported in patients with chronic liver disease, with an estimated risk of thrombosis around 5.5\% [18].

While HIV-associated ITP remains an important clinical problem in the HAART era, the availability of Eltrombopag, gives medical providers additional treatment options. In conclusion, we want to stress that Eltrombopag may be useful for treating severe and refractory HIV-associated ITP patients. Although in our experience neither hepatotoxicity nor thrombotic events were observed, further experience is needed, to better determinate the effectiveness and the safety of Eltrombopag in the context of HIV-associated ITP.

\section{References}

1. Morris L, Distenfeld A, Amorosi E, Karpatkin S (1982) Autoimmune thrombocytopenic purpura in homosexual men. Ann Intern Med 96: 714-717. [Crossref]

2. Walsh CM, Nadri MA, Karpatkin S (1984) On the mechanism of thrombocytopenic purpura in sexually active homosexual men. $N$ Engl J Med 311: 635-639. [Crossref]

3. Mientjes GH, van Ameijden EJ, Mulder JW, van den Hoek JA, Coutinho RA, et al. (1992) Prevalence of thrombocytopenia in HIV-infected and non-HIV infected drug users and homosexual men. Br J Haematol 82: 615-619. [Crossref]

4. Sloand EM, Klein HG, Banks SM, Vareldzis B, Merrin S (1992) Epidemiology of thrombocytopenia in HIV infection. Eur J Haematol 48: 168-172.

5. Servais J, Nkoghe D, Schmit JC (2001) HIV-associated hematologic disorders are correlated with plasma viral load and improve under highly active antiretroviral therapy. J Acquir Immue Defic Syndr 28: 221-225.

6. Marieke Schoonen W, Kucera G, Coalson J (2009) An epidemiology of immune thrombocytopenic purpura in the General Practice Research Database. Br J Haematol 145: 235-244.

7. Marks KM, Clarke RM, Bussel JB (2009) Risk factors for thrombocytopenia in HIVinfected persons in the era of potent antiretroviral therapy. J Acquir Immue Defic Syndr 52: 595-599.

8. Ochoa R, Akunyili I, Milone AM, Harrington TJ, Byrnes JJ (2011) Sustained responses to thrombopoetin analogs in HIV patients with refractory ITP. Abstract presented at: $53^{\text {rd }}$ ASH Annual Meeting and Exposition: December 10-13. San Diego, CA. Abstract 1165.

9. Frederiksen H, Merete LM, Mette N (2014) Twenty-year mortality of adult patients with primary immune thrombocytopenia: a Danish population-based cohort study. $\mathrm{Br}$ J Haematol 166: 260-267.

10. Kowalczyk M, Rubinstein PG, Aboulafia DM (2015) Initial experience with the use of thrombopoetin receptor agonists in patients with refractory HIV-associated immune thrombocytopenic purpura. A case series. J Int Assoc Provid AIDS Care 14: 211-216.

11. Psaila B, Bussel JB (2007) Immune thrombocytopenic Purpura. Hematol Oncol Clin North Am 21: 743-759.

12. The Swiss Group for Clinical Studies on the Acquired Immunodeficiency Syndrome (1998) Zidovudine for the treatment of thrombocytopenia associated with human immunodeficiency virus (HIV). A prospective study. Ann Intern Med 109: 718-721.

13. Scaradavou S (2002) HIV-related thrombocytopenia. Blood Rev 16: 73-76.

14. Rodegheiro F, Stasi R, Giagounidis A (2013) Long-term safety and tolerabilityvof Romiplostim in patients with primary immune thrombocytopenia: a pooled analysis of 13 clinical trials. Eur J Haematol 91: 423-436.

15. Saleh MN, Bussel JB, Cheng G (2013) Safety and efficacy of Eltrombopag for treatment of chronic immune thrombocytopenia: results of the long-term, open-label EXTEND study. Blood 121: 537-545.

16. BrynesRK, Orazi A, Bakshi K, Bailey CK, Chan GW (2013) Evaluation of the effects of long-term treatment with Eltrombopag on bone marrow in patients with chronic immune thrombocytopenia (ITP) data from the EXTEND study. Abstract presented at the $55^{\text {th }}$ ASH Annual Meeting and Exposition; December 7-10. New Orleans, LA. Abstract 326. 
Juárez-Salcedo LM (2020) Successful use of Eltrombopag in a patient with refractory HIV-associated immune thrombocytopenic purpura

17. Bussel JB, Saleh M, Meyer O (2010) Results of bone marrow examinations in patients with chronic idiopathic (immune) thrombocytopenic purpura treated with Eltrombopag for more than one year. Haematologica 95: 253 .
18. Cheng G (2013) Thrombopoietin receptor agonists for the treatment of ITP. Jpn J Clin Hematol 54: 1915-1918. [Crossref]

Copyright: (2020 Juárez-Salcedo LM. This is an open-access article distributed under the terms of the Creative Commons Attribution License, which permits unrestricted use, distribution, and reproduction in any medium, provided the original author and source are credited. 